\title{
SymPy: symbolic computing in Python
}

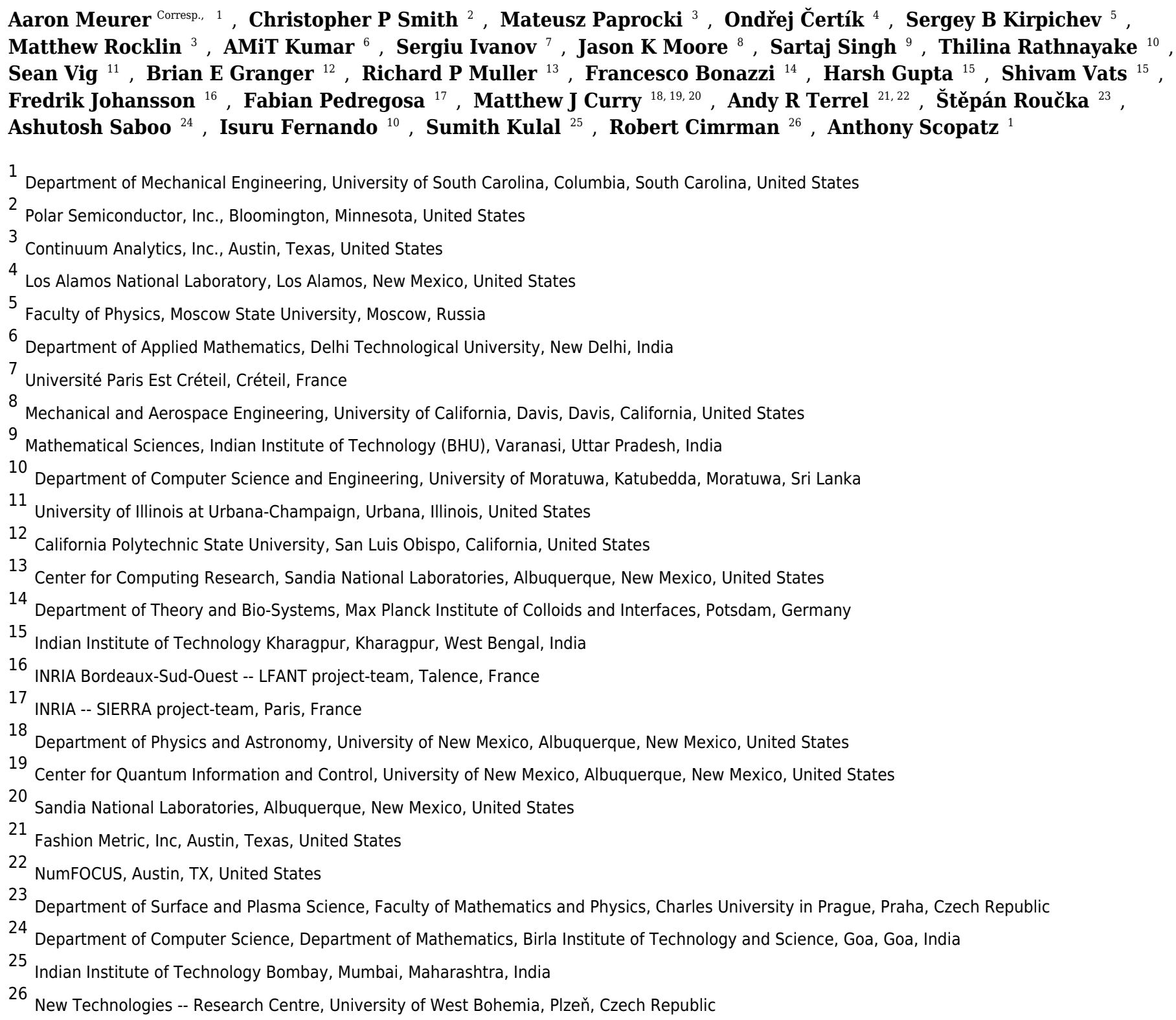

Corresponding Author: Aaron Meurer

Email address: asmeurer@gmail.com

SymPy is an open source computer algebra system written in pure Python. It is built with a focus on extensibility and ease of use, through both interactive and programmatic applications. These characteristics have led SymPy to become a popular symbolic library for the scientific Python ecosystem. This paper presents the architecture of SymPy, a description of its features, and a discussion of select submodules. The supplementary material provide additional examples and further outline details of the architecture and features of SymPy. 


\title{
SymPy: symbolic computing in Python
}

\author{
Aaron Meurer ${ }^{1}$, Christopher P. Smith ${ }^{2}$, Mateusz Paprocki ${ }^{3}$, Ondřej Čertík ${ }^{4}$, \\ Sergey B. Kirpichev ${ }^{5}$, Matthew Rocklin ${ }^{3}$, AMiT Kumar ${ }^{6}$, Sergiu Ivanov ${ }^{7}$, \\ Jason K. Moore ${ }^{8}$, Sartaj Singh ${ }^{9}$, Thilina Rathnayake ${ }^{10}$, Sean Vig ${ }^{11}$, Brian \\ E. Granger ${ }^{12}$, Richard P. Muller ${ }^{13}$, Francesco Bonazzi ${ }^{14}$, Harsh Gupta ${ }^{15}$, \\ Shivam Vats ${ }^{15}$, Fredrik Johansson ${ }^{16}$, Fabian Pedregosa ${ }^{17}$, Matthew J. \\ Curry ${ }^{18,} 19,20$, Andy R. Terrel ${ }^{21,22}$, Štěpán Roučkaa ${ }^{23}$, Ashutosh Saboo ${ }^{24}$, \\ Isuru Fernando ${ }^{10}$, Sumith Kulal ${ }^{25}$, Robert Cimrman ${ }^{26}$, and Anthony \\ Scopatz ${ }^{1}$
}

${ }^{1}$ Department of Mechanical Engineering, University of South Carolina, Columbia, South Carolina, United States

${ }^{2}$ Polar Semiconductor, Inc., Bloomington, Minnesota, United States

${ }^{3}$ Continuum Analytics, Inc., Austin, Texas, United States

${ }^{4}$ Los Alamos National Laboratory, Los Alamos, New Mexico, United States

${ }^{5}$ Faculty of Physics, Moscow State University, Moscow, Russia

${ }^{6}$ Department of Applied Mathematics, Delhi Technological University, New Delhi, India

${ }^{7}$ Université Paris Est Créteil, Créteil, France

${ }^{8}$ Mechanical and Aerospace Engineering, University of California, Davis, Davis,

California, United States

${ }^{9}$ Mathematical Sciences, Indian Institute of Technology (BHU), Varanasi, Uttar Pradesh, India

${ }^{10}$ Department of Computer Science and Engineering, University of Moratuwa, Katubedda, Moratuwa, Sri Lanka

${ }^{11}$ University of Illinois at Urbana-Champaign, Urbana, Illinois, United States

${ }^{12}$ California Polytechnic State University, San Luis Obispo, California, United States

${ }^{13}$ Center for Computing Research, Sandia National Laboratories, Albuquerque, New Mexico, United States

${ }^{14}$ Department of Theory and Bio-Systems, Max Planck Institute of Colloids and Interfaces, Potsdam, Germany

${ }^{15}$ Indian Institute of Technology Kharagpur, Kharagpur, West Bengal, India

${ }^{16}$ INRIA Bordeaux-Sud-Ouest - LFANT project-team, Talence, France

${ }^{17}$ INRIA - SIERRA project-team, Paris, France

${ }^{18}$ Department of Physics and Astronomy, University of New Mexico, Albuquerque, New Mexico, United States

${ }^{19}$ Center for Quantum Information and Control, University of New Mexico, Albuquerque, New Mexico, United States

${ }^{20}$ Sandia National Laboratories, Albuquerque, New Mexico, United States

${ }^{21}$ Fashion Metric, Inc, Austin, Texas, United States

${ }^{22}$ NumFOCUS, Austin, TX, United States

${ }^{23}$ Department of Surface and Plasma Science, Faculty of Mathematics and Physics, Charles University in Prague, Praha, Czech Republic

${ }^{24}$ Department of Computer Science, Department of Mathematics, Birla Institute of

Technology and Science, Goa, Goa, India

${ }^{25}$ Indian Institute of Technology Bombay, Mumbai, Maharashtra, India

${ }^{26}$ New Technologies - Research Centre, University of West Bohemia, PIzeň, Czech

Republic 


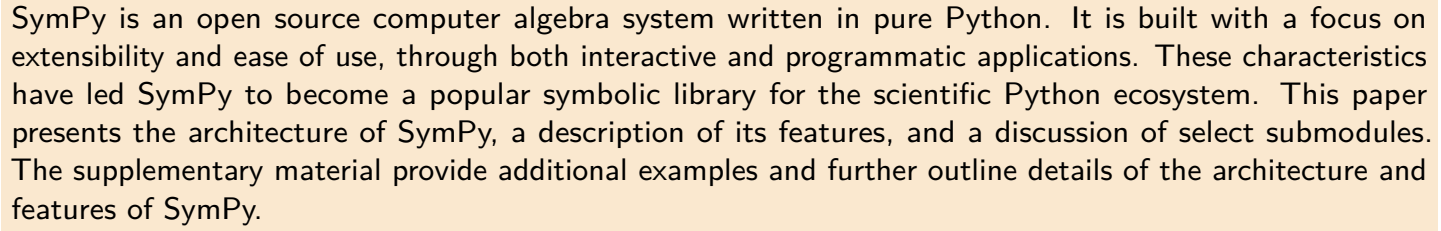

\section{INTRODUCTION}

SymPy is a full featured computer algebra system (CAS) written in the Python [32] programming language. It is free and open source software, licensed under the 3-clause BSD license [49]. The SymPy project was started by Ondřej Čertík in 2005, and it has since grown to over 500 contributors. Currently, SymPy is developed on GitHub using a bazaar community model [43]. The accessibility of the codebase and the open community model allow SymPy to rapidly respond to the needs of users and developers.

Python is a dynamically typed programming language that has a focus on ease of use and readability. ${ }^{1}$ Due in part to this focus, it has become a popular language for scientific computing and data science, with a broad ecosystem of libraries [37]. SymPy is itself used as a dependency by many libraries and tools to support research within a variety of domains, such as SageMath [58] (pure and applied mathematics), yt [64] (astronomy and astrophysics), PyDy [19] (multibody dynamics), and SfePy [10] (finite elements).

Unlike many CAS's, SymPy does not invent its own programming language. Python itself is used both for the internal implementation and end user interaction. By using the operator overloading functionality of Python, SymPy follows the embedded domain specific language paradigm proposed by Hudak [24]. The exclusive usage of a single programming language makes it easier for people already familiar with that language to use or develop SymPy. Simultaneously, it enables developers to focus on mathematics, rather than language design. SymPy version 1.0 officially supports Python 2.6, 2.7 and 3.2-3.5.

SymPy is designed with a strong focus on usability as a library. Extensibility is important in its application program interface (API) design. Thus, SymPy makes no attempt to extend the Python language itself. The goal is for users of SymPy to be able to include SymPy alongside other Python libraries in their workflow, whether that be in an interactive environment or as a programmatic part in a larger system.

Being a library, SymPy does not have a built-in graphical user interface (GUI). However, SymPy exposes a rich interactive display system, and supports registering display formatters with Jupyter [29] frontends, including the Notebook and Qt Console, which will render SymPy expressions using MathJax [9] or $\mathrm{HAT}_{\mathrm{E}} \mathrm{X}$.

The remainder of this paper discusses key components of the SymPy library. Section 2 enumerates the features of SymPy and takes a closer look at some of the important ones. The section 3 looks at the numerical features of SymPy and its dependency library, mpmath. Section 4 looks at the domain specific physics submodules for performing symbolic and numerical calculations in classical mechanics and quantum mechanics. Section 5 discusses the architecture of SymPy. Section 6 looks at a selection of packages that depend on SymPy. Conclusions and future directions for SymPy are given in section 7. All examples in this paper use SymPy version 1.0 and mpmath version 0.19 .

Additionally, the supplementary material takes a deeper look at a few SymPy topics. Supplement section 1 discusses the Gruntz algorithm, which SymPy uses to calculate symbolic limits. Sections 2-9 of the supplement discuss the series, logic, Diophantine equations, sets, statistics, category theory, tensor, and numerical simplification submodules of SymPy, respectively. Supplement section 10 provides additional examples for topics discussed in the main paper. Supplement section 11 discusses the SymPy Gamma project. Finally, section 12 of the supplement contains a

${ }^{1}$ This paper assumes a moderate familiarity with the Python programming language. 
brief comparison of SymPy with Wolfram Mathematica.

The following statement imports all SymPy functions into the global Python namespace. ${ }^{2}$ From here on, all examples in this paper assume that this statement has been executed: ${ }^{3}$

>> from sympy import *

All the examples in this paper can be tested on SymPy Live, an online Python shell that uses the Google App Engine [11] to execute SymPy code. SymPy Live is also integrated into the SymPy documentation at http://docs.sympy.org.

\section{OVERVIEW OF CAPABILITIES}

This section gives a basic introduction of SymPy, and lists its features. A few featuresassumptions, simplification, calculus, polynomials, printers, solvers, and matrices - are core components of SymPy and are discussed in depth. Many other features are discussed in depth in the supplementary material.

\subsection{Basic Usage}

Symbolic variables, called symbols, must be defined and assigned to Python variables before they can be used. This is typically done through the symbols function, which may create multiple symbols in a single function call. For instance,

$\gg x, y, z=\operatorname{symbols}(' x y z$ ')

creates three symbols representing variables named $x, y$, and $z$. In this particular instance, these symbols are all assigned to Python variables of the same name. However, the user is free to assign them to different Python variables, while representing the same symbol, such as a, $b$, $c=\operatorname{symbols}\left({ }^{\prime} x \quad y \quad z^{\prime}\right)$. In order to minimize potential confusion, though, all examples in this paper will assume that the symbols $x, y$, and $z$ have been assigned to Python variables identical to their symbolic names.

Expressions are created from symbols using Python's mathematical syntax. For instance, the following Python code creates the expression $\left(x^{2}-2 x+3\right) / y$. Note that the expression remains unevaluated: it is represented symbolically.

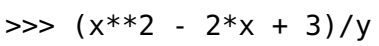

\subsection{List of Features}

Although SymPy's extensive feature set cannot be covered in depth in this paper, bedrock areas, that is, those areas that are used throughout the library, are discussed in their own subsections below. Additionally, Table 1 gives a compact listing of all major capabilities present in the SymPy codebase. This grants a sampling from the breadth of topics and application domains that SymPy services. Unless stated otherwise, all features noted in Table 1 are symbolic in nature. Numeric features are discussed in Section 3.

Table 1. SymPy Features and Descriptions.

\begin{tabular}{l}
\hline Feature (submodules) Description \\
\hline Calculus (sympy.core, \\
sympy.calculus, \\
sympy.integrals, \\
sympy. series) \\
statements in prodithms for computing derivatives, integrals, and limits. \\
imported names could clash with already existing imports from another package. For example, SymPy, the \\
standard Python math library, and NumPy all define the exp function, but only the SymPy one will work with \\
SymPy symbolic expressions. \\
3 The three greater-than signs denote the user input for the Python interactive session, with the result, if \\
there is one, shown on the next line.
\end{tabular}




\author{
Category Theory \\ (sympy.categories) \\ Code Generation \\ (sympy.printing, \\ sympy. codegen) \\ Combinatorics \& Group \\ Theory \\ (sympy.combinatorics) \\ Concrete Math \\ (sympy.concrete)
}

Cryptography

(sympy.crypto)

Differential Geometry

(sympy.diffgeom)

Geometry (sympy.geometry)

Lie Algebras

(sympy . liealgebras)

Logic (sympy. logic)

Matrices (sympy.matrices)

Matrix Expressions (sympy.matrices.expressions) Number Theory

(sympy.ntheory)

Plotting (sympy.plotting)

Polynomials (sympy.polys)

Printing (sympy.printing)

Quantum Mechanics

(sympy.physics.quantum)
Representation of objects, morphisms, and diagrams. Tools for drawing diagrams with Xy-pic [48].

Generation of compilable and executable code in a variety of different programming languages from expressions directly. Target languages include C, Fortran, Julia, JavaScript, Mathematica, MATLAB and Octave, Python, and Theano.

Permutations, combinations, partitions, subsets, various permutation groups (such as polyhedral, Rubik, symmetric, and others), Gray codes [36], and Prufer sequences [4].

Summation, products, tools for determining whether summation and product expressions are convergent, absolutely convergent, hypergeometric, and for determining other properties; computation of Gosper's normal form [42] for two univariate polynomials.

Block and stream ciphers, including shift, Affine, substitution, Vigenère's, Hill's, bifid, RSA, Kid RSA, linear-feedback shift registers, and Elgamal encryption.

Representations of manifolds, metrics, tensor products, and coordinate systems in Riemannian and pseudo-Riemannian geometries [52].

Representations of 2D geometrical entities, such as lines and circles. Enables queries on these entities, such as asking the area of an ellipse, checking for collinearity of a set of points, or finding the intersection between objects.

Representations of Lie algebras and root systems.

Boolean expressions, equivalence testing, satisfiability, and normal forms.

Tools for creating matrices of symbols and expressions. Both sparse and dense representations, as well as symbolic linear algebraic operations (e.g., inversion and factorization), are supported.

Matrices with symbolic dimensions (unspecified entries). Block matrices.

Prime number generation, primality testing, integer factorization, continued fractions, Egyptian fractions, modular arithmetic, quadratic residues, partitions, binomial and multinomial coefficients, prime number tools, hexidecimal digits of $\pi$, and integer factorization.

Hooks for visualizing expressions via matplotlib [25] or as text drawings when lacking a graphical back-end. 2D function plotting, 3D function plotting, and 2D implicit function plotting are supported.

Polynomial algebras over various coefficient domains. Functionality ranges from simple operations (e.g., polynomial division) to advanced computations (e.g., Gröbner bases [1] and multivariate factorization over algebraic number domains).

Functions for printing SymPy expressions in the terminal with ASCII or Unicode characters and converting SymPy expressions to IATEX and MathML.

Quantum states, bra-ket notation, operators, basis sets, representations, tensor products, inner products, outer products, commutators, anticommutators, and specific quantum system implementations. 
Series (sympy.series)

Sets (sympy.sets)

Simplification

(sympy.simplify)

Solvers (sympy.solvers)

Special Functions

(sympy.functions)

Statistics (sympy.stats)

Tensors (sympy.tensor)

Vectors (sympy.vector)
Series expansion, sequences, and limits of sequences. This includes Taylor, Laurent, and Puiseux series as well as special series, such as Fourier and formal power series.

Representations of empty, finite, and infinite sets (including special sets such as the natural, integer, and complex numbers). Operations on sets such as union, intersection, Cartesian product, and building sets from other sets are supported.

Functions for manipulating and simplifying expressions. Includes algorithms for simplifying hypergeometric functions, trigonometric expressions, rational functions, combinatorial functions, square root denesting, and common subexpression elimination.

Functions for symbolically solving equations, systems of equations, both linear and non-linear, inequalities, ordinary differential equations, partial differential equations, Diophantine equations, and recurrence relations.

Implementations of a number of well known special functions, including Dirac delta, Gamma, Beta, Gauss error functions, Fresnel integrals, Exponential integrals, Logarithmic integrals, Trigonometric integrals, Bessel, Hankel, Airy, B-spline, Riemann Zeta, Dirichlet eta, polylogarithm, Lerch transcendent, hypergeometric, elliptic integrals, Mathieu, Jacobi polynomials, Gegenbauer polynomial, Chebyshev polynomial, Legendre polynomial, Hermite polynomial, Laguerre polynomial, and spherical harmonic functions.

Support for a random variable type as well as the ability to declare this variable from prebuilt distribution functions such as Normal, Exponential, Coin, Die, and other custom distributions [47].

Symbolic manipulation of indexed objects.

Basic operations on vectors and differential calculus with respect to $3 \mathrm{D}$ Cartesian coordinate systems.

\subsection{Assumptions}

The assumptions system allows users to specify that symbols have certain common mathematical properties, such as being positive, imaginary, or integer. SymPy is careful to never perform simplifications on an expression unless the assumptions allow them. For instance, the simplification $\sqrt{t^{2}}=t$ holds if $t$ is nonnegative $(t \geq 0)$, but it does not hold for a general complex $t .{ }^{4}$

By default, SymPy performs all calculations assuming that symbols are complex valued. This assumption makes it easier to treat mathematical problems in full generality.

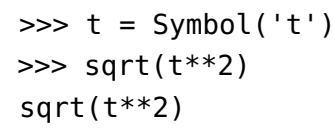

By assuming the most general case, that $t$ is complex by default, SymPy avoids performing mathematically invalid operations. However, in many cases users will wish to simplify expressions containing terms like $\sqrt{t^{2}}$.

Assumptions are set on Symbol objects when they are created. For instance Symbol(' $t$ ', positive=True) will create a symbol named $t$ that is assumed to be positive.

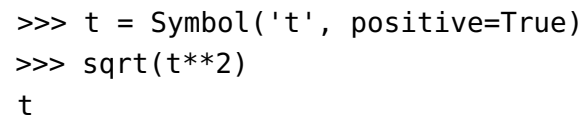

${ }^{4}$ In SymPy, $\sqrt{z}$ is defined on the usual principal branch with the branch cut along the negative real axis. 
Some of the common assumptions are negative, real, nonpositive, integer, prime and commutative. ${ }^{5}$ Assumptions on any SymPy object can be checked with the is assumption attributes, like t.is positive.

Assumptions are only needed to restrict a domain so that certain simplifications can be performed. They are not required to make the domain match the input of a function. For instance, one can create the object $\sum_{n=0}^{m} f(n)$ as $\operatorname{Sum}(f(n),(n, 0, m))$ without setting integer=True when creating the Symbol object $\mathrm{n}$.

The assumptions system additionally has deductive capabilities. The assumptions use a three-valued logic using the Python built in objects True, False, and None. Note that False is returned if the SymPy object doesn't or can't have the assumption. For example, both I.is_real and I.is prime return False for the imaginary unit I.

None represents the "unknown" case. This could mean that given assumptions do not unambiguously specify the truth of an attribute. For instance, Symbol (' $x$ ' , real=True).is_positive will give None because a real symbol might be positive or negative. None could also mean that not enough is known or implemented to compute the given fact. For instance, $(p i+E)$. is_irrational gives None - indeed, the rationality of $\pi+e$ is an open problem in mathematics [31].

Basic implications between the facts are used to deduce assumptions. Deductions are made using the Rete algorithm [13]. ${ }^{6}$ For instance, the assumptions system knows that being an integer implies being rational.

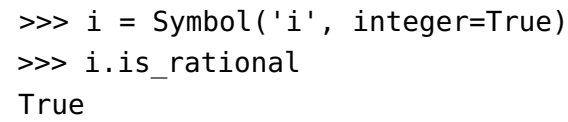

Furthermore, expressions compute the assumptions on themselves based on the assumptions of their arguments. For instance, if $x$ and $y$ are both created with positive=True, then $(x+$ $y)$.is_positive will be True (whereas $(x-y)$.is_positive will be None).

\subsection{Simplification}

The generic way to simplify an expression is by calling the simplify function. It must be emphasized that simplification is not a rigorously defined mathematical operation [34]. The simplify function applies several simplification routines along with heuristics to make the output expression "simple".

It is often preferable to apply more directed simplification functions. These apply very specific rules to the input expression and are typically able to make guarantees about the output. For instance, the factor function, given a polynomial with rational coefficients in several variables, is guaranteed to produce a factorization into irreducible factors. Table 2 lists common simplification functions.

Examples for these simplification functions can be found in section 10 of the supplementary material.

\subsection{Calculus}

SymPy provides all the basic operations of calculus, such as calculating limits, derivatives, integrals, or summations.

Limits are computed with the limit function, using the Gruntz algorithm [22] for computing symbolic limits and heuristics (a description of the Gruntz algorithm may be found in section 1

\footnotetext{
${ }^{5}$ SymPy assumes that two expressions $A$ and $B$ commute with each other multiplicatively, that is, $A \cdot B=B \cdot A$, unless they both have commutative=False. Many algorithms in SymPy require special consideration to work correctly with noncommutative products.

${ }^{6}$ For historical reasons, this algorithm is distinct from the sympy.logic submodule, which is discussed in section 3 of the supplementary material. SymPy also has an experimental assumptions system which stores facts separate from objects, and uses sympy.logic and a SAT solver for deduction. We will not discuss this system here.

${ }^{7}$ The measure parameter of the simplify function lets the user specify the Python function used to determine how complex an expression is. The default measure function returns the total number of operations in the expression
} 
Table 2. Some SymPy Simplification Functions

\begin{tabular}{ll}
\hline expand & expand the expression \\
factor & factor a polynomial into irreducibles \\
collect & collect polynomial coefficients \\
cancel & rewrite a rational function as $p / q$ with common factors canceled \\
apart & compute the partial fraction decomposition of a rational function \\
trigsimp & simplify trigonometric expressions $[18]$ \\
hyperexpand & expand hypergeometric functions $[44,45]$ \\
\hline
\end{tabular}

of the supplementary material). For example, the following computes $\lim _{x \rightarrow \infty} x \sin \left(\frac{1}{x}\right)=1$. Note that SymPy denotes $\infty$ as oo (two lower case "o"s).

$\gg>\operatorname{limit}\left(x^{*} \sin (1 / x), x, 00\right)$

1

As a more complex example, SymPy computes

$$
\begin{aligned}
& \lim _{x \rightarrow 0}\left(2 e^{\frac{1-\cos (x)}{\sin (x)}}-1\right)^{\frac{\sinh (x)}{\operatorname{atan}^{2}(x)}}=e . \\
& \text { >> } \operatorname{limit}((2 * \exp ((1-\cos (x)) / \sin (x))-1) * *(\sinh (x) / \operatorname{atan}(x) * * 2), x, 0) \\
& \text { P }
\end{aligned}
$$

Derivatives are computed with the diff function, which recursively uses the various differentiation rules.

$\gg>\operatorname{diff}(\sin (x) * \exp (x), x)$

$\exp (x) * \sin (x)+\exp (x) * \cos (x)$

Integrals are calculated with the integrate function. SymPy implements a combination of the Risch algorithm [7], table lookups, a reimplementation of Manuel Bronstein's "Poor Man's Integrator" [6], and an algorithm for computing integrals based on Meijer G-functions [44, 45]. These allow SymPy to compute a wide variety of indefinite and definite integrals. The Meijer G-function algorithm and the Risch algorithm are respectively demonstrated below by the computation of

$$
\int_{0}^{\infty} e^{-s t} \log (t) d t=-\frac{\log (s)+\gamma}{s}
$$

and

$$
\int \frac{-2 x^{2}(\log (x)+1) e^{x^{2}}+\left(e^{x^{2}}+1\right)^{2}}{x\left(e^{x^{2}}+1\right)^{2}(\log (x)+1)} d x=\log (\log (x)+1)+\frac{1}{e^{x^{2}+1}} .
$$

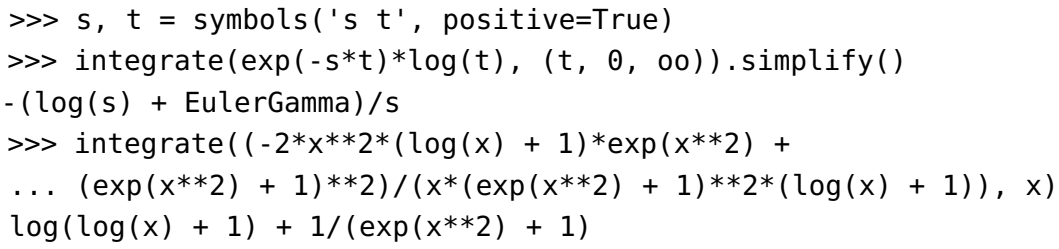

Summations are computed with the summation function, which uses a combination of Gosper's algorithm [21], an algorithm that uses Meijer G-functions [44, 45], and heuristics. Products are computed with product function via a suite of heuristics. 


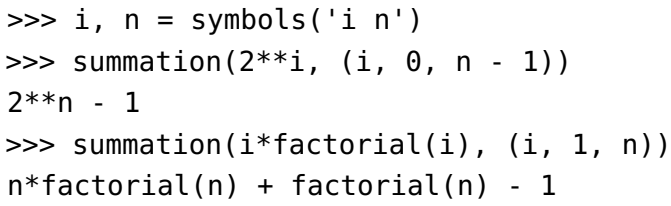

Series expansions are computed with the series function. This example computes the power series of $\sin (x)$ around $x=0$ up to $x^{6}$.

$\gg>\operatorname{series}(\sin (x), x, 0,6)$

$\mathrm{x}-\mathrm{x} * 3 / 6+\mathrm{x} * * 5 / 120+0(\mathrm{x} * * 6)$

Section 2 of the supplementary material discusses series expansions methods in more depth. Integrals, derivatives, summations, products, and limits that cannot be computed return unevaluated objects. These can also be created directly if the user chooses.

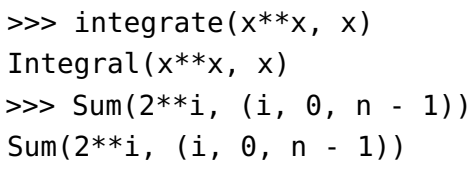

\subsection{Polynomials}

SymPy implements a suite of algorithms for polynomial manipulation, which ranges from relatively simple algorithms for doing arithmetic of polynomials, to advanced methods for factoring multivariate polynomials into irreducibles, symbolically determining real and complex root isolation intervals, or computing Gröbner bases.

Polynomial manipulation is useful in its own right. Within SymPy, though, it is mostly used indirectly as a tool in other areas of the library. In fact, many mathematical problems in symbolic computing are first expressed using entities from the symbolic core, preprocessed, and then transformed into a problem in the polynomial algebra, where generic and efficient algorithms are used to solve the problem. The solutions to the original problem are subsequently recovered from the results. This is a common scheme in symbolic integration or summation algorithms.

SymPy implements dense and sparse polynomial representations. ${ }^{8}$ Both are used in the univariate and multivariate cases. The dense representation is the default for univariate polynomials. For multivariate polynomials, the choice of representation is based on the application. The most common case for the sparse representation is algorithms for computing Gröbner bases (Buchberger, F4, and F5) $[8,14,15]$. This is because different monomial orderings can be expressed easily in this representation. However, algorithms for computing multivariate GCDs or factorizations, at least those currently implemented in SymPy [38], are better expressed when the representation is dense. The dense multivariate representation is specifically a recursively-dense representation, where polynomials in $K\left[x_{0}, x_{1}, \ldots, x_{n}\right]$ are viewed as a polynomials in $K\left[x_{0}\right]\left[x_{1}\right] \ldots\left[x_{n}\right]$. Note that despite this, the coefficient domain $K$, can be a multivariate polynomial domain as well. The dense recursive representation in Python gets inefficient as the number of variables increases.

Some examples for the sympy. polys submodule can be found in section 10 of the supplementary material.

\subsection{Printers}

SymPy has a rich collection of expression printers. By default, an interactive Python session will render the str form of an expression, which has been used in all the examples in this paper so far. The str form of an expression is valid Python and roughly matches what a user would type to enter the expression. ${ }^{9}$

\footnotetext{
${ }^{8}$ In a dense representation, the coefficients for all terms up to the degree of each variable are stored in memory In a sparse representation, only the nonzero coefficients are stored.

${ }^{9}$ Many Python libraries distinguish the str form of an object, which is meant to be human-readable, and the repr form, which is mean to be valid Python that recreates the object. In SymPy, str(expr) =e repr(expr). In other words, the string representation of an expression is designed to be compact, human-readable, and valid Python code that could be used to recreate the expression. As noted in section 5.1, the srepr function prints the exact, verbose form of an expression.
} 


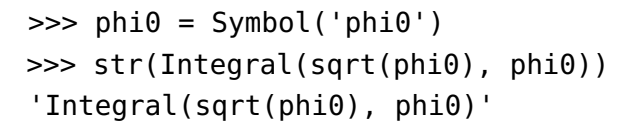

A two-dimensional (2D) textual representation of the expression can be printed with monospace fonts via pprint. Unicode characters are used for rendering mathematical symbols such as integral signs, square roots, and parentheses. Greek letters and subscripts in symbol names that have Unicode code points associated are also rendered automatically.

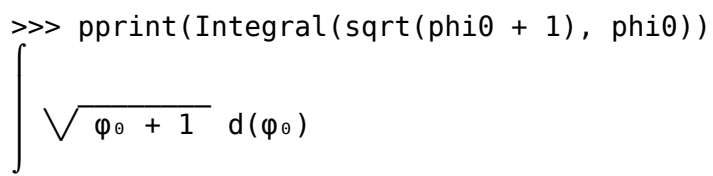

Alternately, the use_unicode=False flag can be set, which causes the expression to be printed using only ASCII characters.

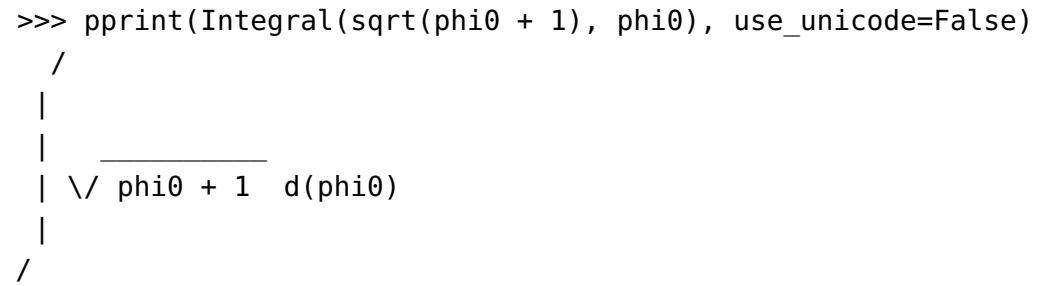

The function latex returns a $\mathrm{LAT}_{\mathrm{E}} \mathrm{X}$ representation of an expression.

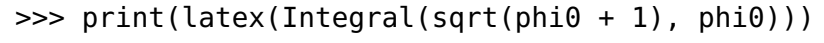

Users are encouraged to run the init printing function at the beginning of interactive sessions, which automatically enables the best pretty printing supported by their environment. In the Jupyter Notebook or Qt Console [40], the $\mathrm{LAT}_{\mathrm{E}} \mathrm{X}$ printer is used to render expressions using MathJax or $\mathrm{AT}_{\mathrm{E}} \mathrm{X}$, if it is installed on the system. The 2D text representation is used otherwise.

Other printers such as MathML are also available. SymPy uses an extensible printer subsystem, which allows extending any given printer, and also allows custom objects to define their printing behavior for any printer. The code generation functionality of SymPy relies on this subsystem to convert expressions into code in various target programming languages.

\subsection{Solvers}

SymPy has equation solvers that can handle ordinary differential equations, recurrence relationships, Diophantine equations ${ }^{10}$, and algebraic equations. There is also rudimentary support for simple partial differential equations.

There are two functions for solving algebraic equations in SymPy: solve and solveset. solveset has several design changes with respect to the older solve function. This distinction is present in order to resolve the usability issues with the previous solve function API while maintaining backward compatibility with earlier versions of SymPy. solveset only requires essential input information from the user. The function signatures of solve and solveset are

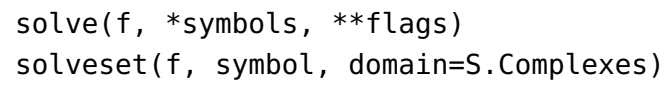

The domain parameter can be any set from the sympy. sets module (see section 5 of the supplementary material for details on sympy. sets), but is typically either S.Complexes (the default) or S.Reals; the latter causes solveset to only return real solutions.

\footnotetext{
${ }^{10}$ See section 4 of the supplementary material for an in depth discussion on the Diophantine submodule.
} 
An important difference between the two functions is that the output API of solve varies with input (sometimes returning a Python list and sometimes a Python dictionary) whereas solveset always returns a SymPy set object.

Both functions implicitly assume that expressions are equal to 0 . For instance, solveset ( $x$ $1, \mathrm{x}$ ) solves $x-1=0$ for $x$.

solveset is under active development as a planned replacement for solve. There are certain features which are implemented in solve that are not yet implemented in solveset, including multivariate systems, and some transcendental equations.

Some examples for solveset and solve can be found in section 10 of the supplementary material.

\subsection{Matrices}

Besides being an important feature in its own right, computations on matrices with symbolic entries are important for many algorithms within SymPy. The following code shows some basic usage of the Matrix class.

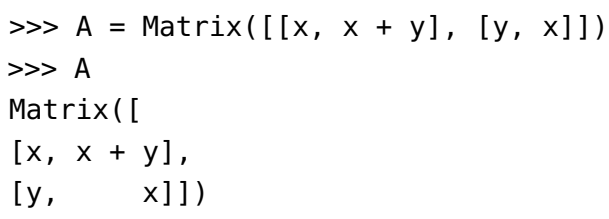

SymPy matrices support common symbolic linear algebra manipulations, including matrix addition, multiplication, exponentiation, computing determinants, solving linear systems, singular values, and computing inverses using LU decomposition, LDL decomposition, Gauss-Jordan elimination, Cholesky decomposition, Moore-Penrose pseudoinverse, or adjugate matrices.

All operations are performed symbolically. For instance, eigenvalues are computed by generating the characteristic polynomial using the Berkowitz algorithm and then finding its zeros using polynomial routines.

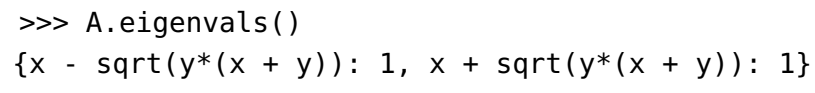

Internally these matrices store the elements as Lists of Lists (LIL) [27], meaning the matrix is stored as a list of lists of entries (effectively, the input format used to create the matrix A above), making it a dense representation. ${ }^{11}$ For storing sparse matrices, the SparseMatrix class can be used. Sparse matrices store their elements in Dictionary of Keys (DOK) format, meaning that the entries are stored as a dict of (row, column) pairs mapping to the elements.

SymPy also supports matrices with symbolic dimension values. Matrixsymbol represents a matrix with dimensions $m \times n$, where $m$ and $n$ can be symbolic. Matrix addition and multiplication, scalar operations, matrix inverse, and transpose are stored symbolically as matrix expressions.

Block matrices are also implemented in SymPy. BlockMatrix elements can be any matrix expression, including explicit matrices, matrix symbols, and other block matrices. All functionalities of matrix expressions are also present in BlockMatrix.

When symbolic matrices are combined with the assumptions submodule for logical inference, they provide powerful reasoning over invertibility, semi-definiteness, orthogonality, etc., which are valuable in the construction of numerical linear algebra systems [46].

More examples for Matrix and BlockMatrix may be found in section 10 of the supplementary material.

\section{NUMERICS}

While SymPy primarily focuses on symbolics, it is impossible to have a complete symbolic system without the ability to numerically evaluate expressions. Many operations directly use numerical

\footnotetext{
${ }^{11}$ Similar to the polynomials submodule, dense here means that all entries are stored in memory, contrasted with a sparse representation where only nonzero entries are stored.
} 
evaluation, such as plotting a function, or solving an equation numerically. Beyond this, certain purely symbolic operations require numerical evaluation to effectively compute. For instance, determining the truth value of $e+1>\pi$ is most conveniently done by numerically evaluating both sides of the inequality and checking which is larger.

\subsection{Floating-Point Numbers}

Floating-point numbers in SymPy are implemented by the Float class, which represents an arbitrary-precision binary floating-point number by storing its value and precision (in bits). This representation is distinct from the Python built-in float type, which is a wrapper around machine double types and uses a fixed precision (53-bit).

Because Python float literals are limited in precision, strings should be used to input precise decimal values:

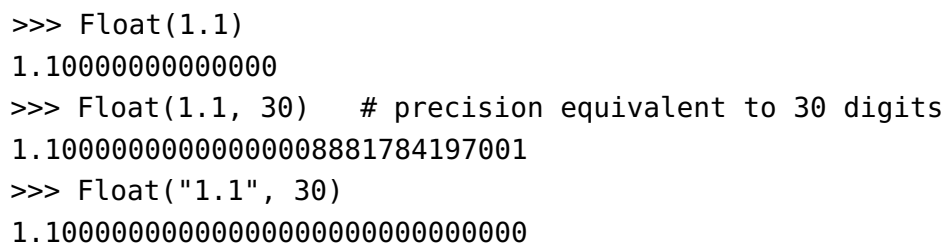

The evalf method converts a constant symbolic expression to a Float with the specified precision, here 25 digits:

>> (pi + 1).evalf $(25)$

4.141592653589793238462643

Float numbers do not track their accuracy, and should be used with caution within symbolic expressions since familiar dangers of floating-point arithmetic apply [20]. A notorious case is that of catastrophic cancellation:

$\gg>\cos (\exp (-100)) \cdot \operatorname{evalf}(25)-1$

0

Applying the evalf method to the whole expression solves this problem. Internally, evalf estimates the number of accurate bits of the floating-point approximation for each sub-expression, and adaptively increases the working precision until the estimated accuracy of the final result matches the sought number of decimal digits:

>> $(\cos (\exp (-100))-1) \cdot \operatorname{evalf}(25)$

$-6.919482633683687653243407 e-88$

The evalf method works with complex numbers and supports more complicated expressions, such as special functions, infinite series, and integrals. The internal error tracking does not provide rigorous error bounds (in the sense of interval arithmetic) and cannot be used to accurately track uncertainty in measurement data; the sole purpose is to mitigate loss of accuracy that typically occurs when converting symbolic expressions to numerical values.

\subsection{The mpmath Library}

The implementation of arbitrary-precision floating-point arithmetic is supplied by the mpmath library [26]. Originally, it was developed as a SymPy submodule but has subsequently been moved to a standalone pure-Python package. The basic datatypes in mpmath are $\mathrm{mpf}$ and mpc, which respectively act as multiprecision substitutes for Python's float and complex. The floating-point precision is controlled by a global context:

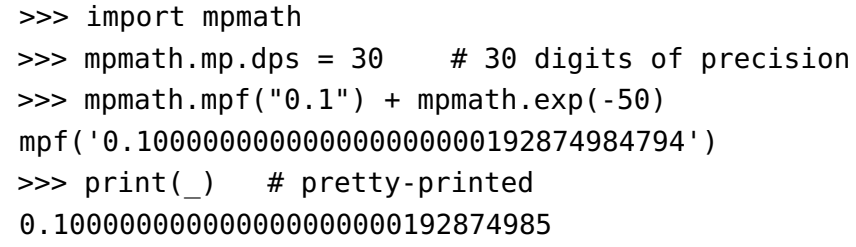


Like SymPy, mpmath is a pure Python library. A design decision of SymPy is to keep it and its required dependencies pure Python. This is a primary advantage of mpmath over other multiple precision libraries such as GNU MPFR [17], which is faster. Like SymPy, mpmath is also BSD licensed (GNU MPFR is licensed under the GNU Lesser General Public License [49]).

Internally, mpmath represents a floating-point number $(-1)^{s} x \cdot 2^{y}$ by a tuple $(s, x, y, b)$ where $x$ and $y$ are arbitrary-size Python integers and the redundant integer $b$ stores the bit length of $x$ for quick access. If GMPY [23] is installed, mpmath automatically uses the gmpy.mpz type for $x$, and GMPY methods for rounding-related operations, improving performance.

Most mpmath and SymPy functions use the same naming scheme, although this is not true in every case. For example, the symbolic SymPy summation expression $\operatorname{Sum}(f(x),(x, a, b))$ representing $\sum_{x=a}^{b} f(x)$ is represented in mpmath as $\operatorname{nsum}(\mathrm{f},(\mathrm{a}, \mathrm{b}))$, where $\mathrm{f}$ is a numeric Python function.

The mpmath library supports special functions, root-finding, linear algebra, polynomial approximation, and numerical computation of limits, derivatives, integrals, infinite series, and solving ODEs. All features work in arbitrary precision and use algorithms that allow computing hundreds of digits rapidly (except in degenerate cases).

The double exponential (tanh-sinh) quadrature is used for numerical integration by default. For smooth integrands, this algorithm usually converges extremely rapidly, even when the integration interval is infinite or singularities are present at the endpoints $[54,2]$. However, for good performance, singularities in the middle of the interval must be specified by the user. To evaluate slowly converging limits and infinite series, mpmath automatically tries Richardson extrapolation and the Shanks transformation (Euler-Maclaurin summation can also be used) [3]. A function to evaluate oscillatory integrals by means of convergence acceleration is also available.

A wide array of higher mathematical functions is implemented with full support for complex values of all parameters and arguments, including complete and incomplete gamma functions, Bessel functions, orthogonal polynomials, elliptic functions and integrals, zeta and polylogarithm functions, the generalized hypergeometric function, and the Meijer G-function. The Meijer G-function instance $G_{1,3}^{3,0}\left(0 ; \frac{1}{2},-1,-\frac{3}{2} \mid x\right)$ is a good test case [63]; past versions of both Maple and Mathematica produced incorrect numerical values for large $x>0$. Here, mpmath automatically removes an internal singularity and compensates for cancellations (amounting to 656 bits of precision when $x=10000$ ), giving correct values:

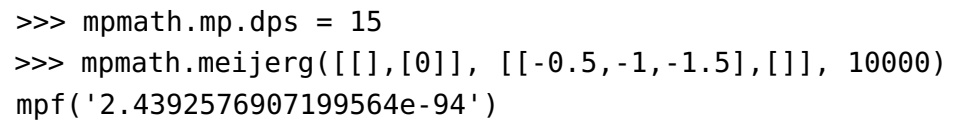

Equivalently, with SymPy's interface this function can be evaluated as:

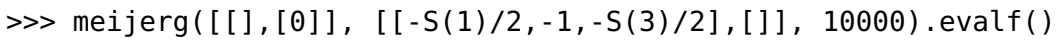

Symbolic integration and summation often produce hypergeometric and Meijer G-function closed forms (see section 2.5); numerical evaluation of such special functions is a useful complement to direct numerical integration and summation.

\section{PHYSICS SUBMODULE}

SymPy includes several submodules that allow users to solve domain specific physics problems. For example, a comprehensive physics submodule is included that is useful for solving problems in mechanics, optics, and quantum mechanics along with support for manipulating physical quantities with units.

\subsection{Classical Mechanics}

One of the core domains that SymPy suports is the physics of classical mechanics. This is in turn separated into two distinct components: vector algebra and mechanics. 


\subsubsection{Vector Algebra}

The sympy. physics.vector submodule provides reference frame-, time-, and space-aware vector and dyadic objects that allow for three-dimensional operations such as addition, subtraction, scalar multiplication, inner and outer products, and cross products. The vector and dyadic objects both can be written in very compact notation that make it easy to express the vectors and dyadics in terms of multiple reference frames with arbitrarily defined relative orientations. The vectors are used to specify the positions, velocities, and accelerations of points; orientations, angular velocities, and angular accelerations of reference frames; and forces and torques. The dyadics are essentially reference frame-aware $3 \times 3$ tensors [53]. The vector and dyadic objects can be used for any one-, two-, or three-dimensional vector algebra, and they provide a strong framework for building physics and engineering tools.

The following Python code demonstrates how a vector is created using the orthogonal unit vectors of three reference frames that are oriented with respect to each other, and the result of expressing the vector in the $A$ frame. The $B$ frame is oriented with respect to the $A$ frame using Z-X-Z Euler Angles of magnitude $\pi, \frac{\pi}{2}$, and $\frac{\pi}{3}$, respectively, whereas the $C$ frame is oriented with respect to the $B$ frame through a simple rotation about the $B$ frame's $X$ unit vector through $\frac{\pi}{2}$.

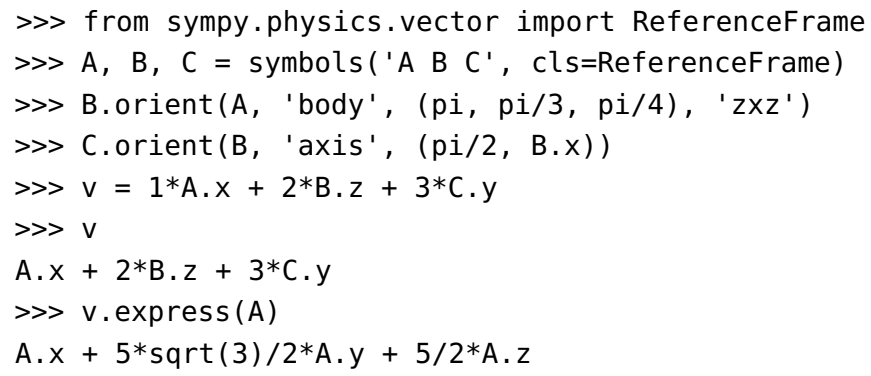

\subsubsection{Mechanics}

The sympy. physics.mechanics submodule utilizes the sympy. physics.vector submodule to populate time-aware particle and rigid-body objects to fully describe the kinematics and kinetics of a rigid multi-body system. These objects store all of the information needed to derive the ordinary differential or differential algebraic equations that govern the motion of the system, i.e., the equations of motion. These equations of motion abide by Newton's laws of motion and can handle arbitrary kinematic constraints or complex loads. The submodule offers two automated methods for formulating the equations of motion based on Lagrangian Dynamics [30] and Kane's Method [28]. Lastly, there are automated linearization routines for constrained dynamical systems [41].

\subsection{Quantum Mechanics}

The sympy. physics .quantum submodule has extensive capabilities to solve problems in quantum mechanics, using Python objects to represent the different mathematical objects relevant in quantum theory [50]: states (bras and kets), operators (unitary, Hermitian, etc.), and basis sets, as well as operations on these objects such as representations, tensor products, inner products, outer products, commutators, and anticommutators. The base objects are designed in the most general way possible to enable any particular quantum system to be implemented by subclassing the base operators and defining the relevant class methods to provide system-specific logic.

Symbolic quantum operators and states may be defined, and one can perform a full range of operations with them.

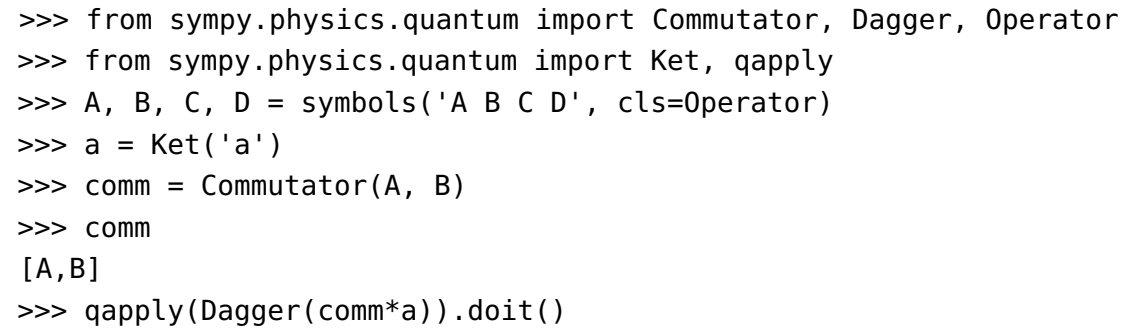


$-<a \mid *(\operatorname{Dagger}(\mathrm{A}) * \operatorname{Dagger}(\mathrm{B}) \quad-\operatorname{Dagger}(\mathrm{B}) * \operatorname{Dagger}(\mathrm{A}))$

Commutators can be expanded using common commutator identities:

$\gg>$ Commutator $(C+B, A * D)$. expand(commutator=True)

$-[A, B] * D-[A, C] * D+A *[B, D]+A *[C, D]$

On top of this set of base objects, a number of specific quantum systems have been implemented in a fully symbolic framework. These include:

- Many of the exactly solvable quantum systems, including simple harmonic oscillator states and raising/lowering operators, infinite square well states, and 3D position and momentum operators and states.

- Second quantized formalism of non-relativistic many-body quantum mechanics [16].

- Quantum angular momentum [65]. Spin operators and their eigenstates can be represented in any basis and for any quantum numbers. A rotation operator representing the Wigner D-matrix, which may be defined symbolically or numerically, is also implemented to rotate spin eigenstates. Functionality for coupling and uncoupling of arbitrary spin eigenstates is provided, including symbolic representations of Clebsch-Gordon coefficients and Wigner symbols.

- Quantum information and computing [35]. Multidimensional qubit states, and a full set of one- and two-qubit gates are provided and can be represented symbolically or as matrices/vectors. With these building blocks, it is possible to implement a number of basic quantum algorithms including the quantum Fourier transform, quantum error correction, quantum teleportation, Grover's algorithm, dense coding, etc. In addition, any quantum circuit may be plotted using the circuit_plot function (Figure 1).

Here are a few short examples of the quantum information and computing capabilities in sympy. physics .quantum. Start with a simple four-qubit state and flip the second qubit from the right using a Pauli-X gate:

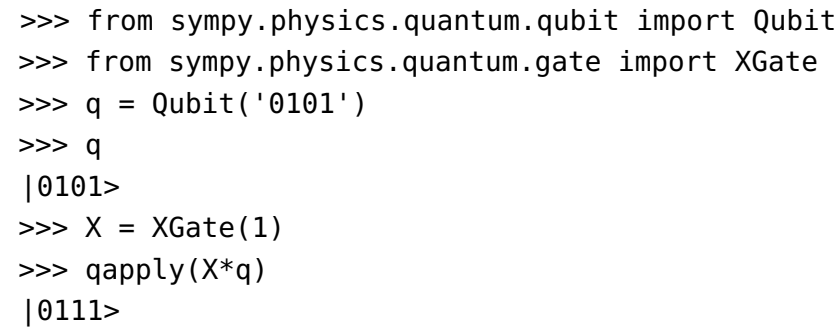

Qubit states can also be used in adjoint operations, tensor products, inner/outer products:

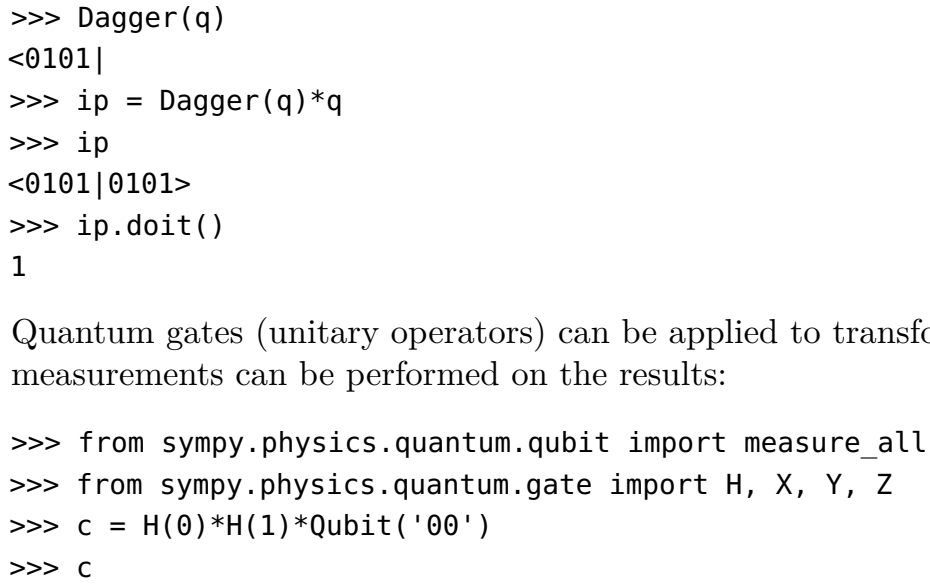
measurements can be performed on the results:

Quantum gates (unitary operators) can be applied to transform these states and then classical 

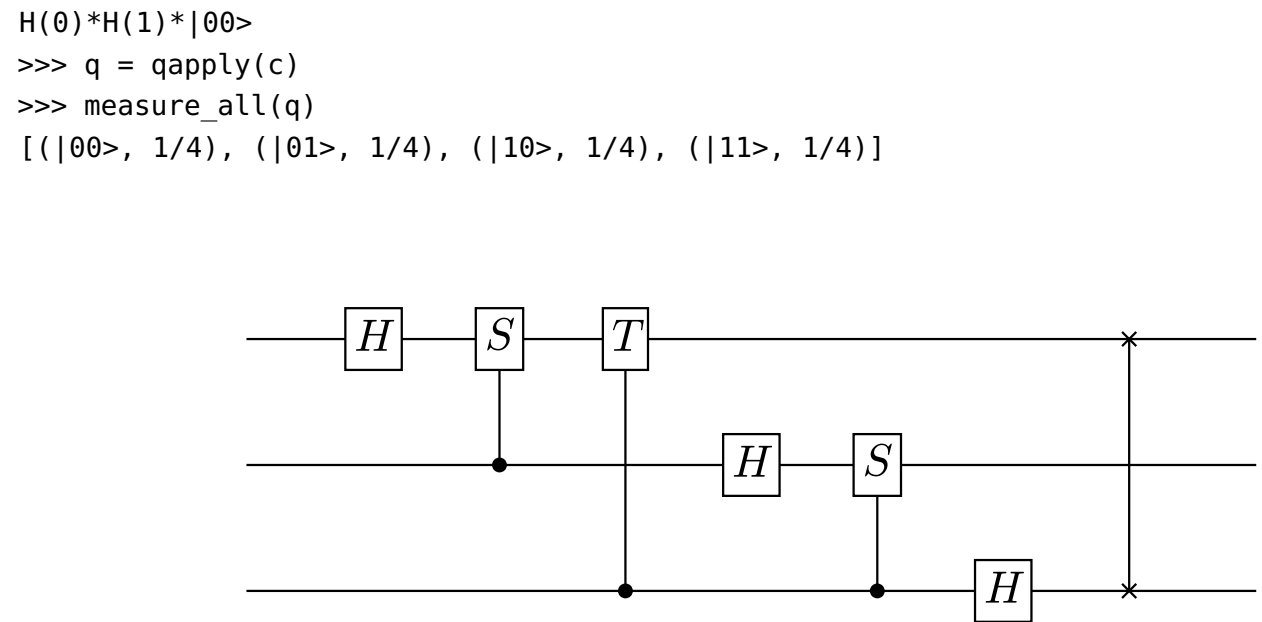

Figure 1. The circuit diagram for a three-qubit quantum Fourier transform generated by SymPy.

Lastly, the following example demonstrates creating a three-qubit quantum Fourier transform, decomposing it into one- and two-qubit gates, and then generating a circuit plot for the sequence of gates (see Figure 1).

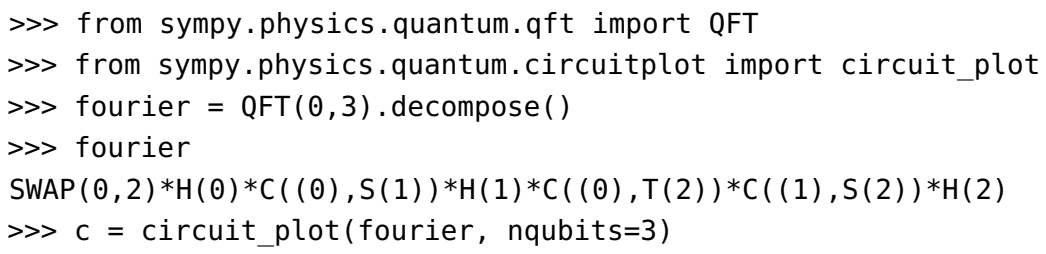

\section{ARCHITECTURE}

Software architecture is of central importance in any large software project because it establishes predictable patterns of usage and development [51]. This section describes the essential structural components of SymPy, provides justifications for the design decisions that have been made, and gives example user-facing code as appropriate.

\subsection{The Core}

A computer algebra system stores mathematical expressions as data structures. For example, the mathematical expression $x+y$ is represented as a tree with three nodes,,$+ x$, and $y$, where $x$ and $y$ are ordered children of + . As users manipulate mathematical expressions with traditional mathematical syntax, the CAS manipulates the underlying data structures. Symbolic computations such as integration, simplification, etc. are all functions that consume and produce expression trees.

In SymPy every symbolic expression is an instance of the class Basic, ${ }^{12}$ the superclass of all SymPy types providing common methods to all SymPy tree-elements, such as traversals. The children of a node in the tree are held in the args attribute. A leaf node in the expression tree has empty args.

For example, consider the expression $x y+2$ :

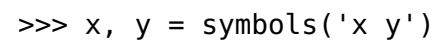

${ }^{12}$ Some internal classes, such as those used in the polynomial submodule, do not follow this rule for efficiency reasons. 
By order of operations, the parent of the expression tree for expr is an addition. It is of type Add. The child nodes of expr are 2 and $x^{*} y$.

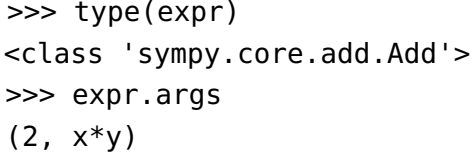

Descending further down into the expression tree yields the full expression. For example, the next child node (given by expr.args[0]) is 2. Its class is Integer, and it has an empty args tuple, indicating that it is a leaf node.

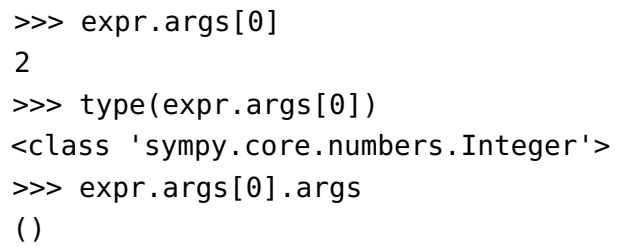

Symbols or symbolic constants, like $e$ or $\pi$, are other examples of leaf nodes.

A useful way to view an expression tree is using the srepr function, which returns a string representation of an expression as valid Python code ${ }^{13}$ with all the nested class constructor calls to create the given expression.

$\gg$ srepr(expr)

"Add(Mul (Symbol ('x'), Symbol('y')), Integer(2))"

Every SymPy expression satisfies a key identity invariant:

expr.func $(* \exp r$.args $)==\exp r$

This means that expressions are rebuildable from their args. ${ }^{14}$ Note that in SymPy the $==$ operator represents exact structural equality, not mathematical equality. This allows testing if any two expressions are equal to one another as expression trees. For example, even though $(x+1)^{2}$ and $x^{2}+2 x+1$ are equal mathematically, SymPy gives

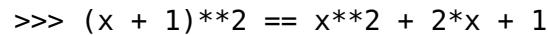

because they are different as expression trees (the former is a Pow object and the latter is an Add object).

Another important property of SymPy expressions is that they are immutable. This simplifies the design of SymPy, and enables expression interning. It also enables expressions to be hashed, which allows expressions to be used as keys in Python dictionaries, and is used to implement caching in SymPy.

Python allows classes to override mathematical operators. The Python interpreter translates the above $x^{*} y+2$ to, roughly, ( $x$._mul_(y))._add_(2). Both $x$ and $y$, returned from the symbols function, are Symbol instances. The 2 in the expression is processed by Python as a

13 The dotprint function from the sympy.printing.dot submodule prints output to dot format, which can be rendered with Graphviz to visualize expression trees graphically.

${ }^{14}$ expr.func is used instead of type(expr) to allow the function of an expression to be distinct from its actual Python class. In most cases the two are the same. 
622

\footnotetext{
${ }^{15}$ See section 3 of the supplementary material for more information on the sympy. logic submodule.
}

literal, and is stored as Python's built in int type. When 2 is passed to the add method of Symbol, it is converted to the SymPy type Integer(2) before being stored in the resulting expression tree. In this way, SymPy expressions can be built in the natural way using Python operators and numeric literals.

\subsection{Extensibility}

While the core of SymPy is relatively small, it has been extended to a wide variety of domains by a broad range of contributors. This is due, in part, to the fact that the same language, Python, is used both for the internal implementation and the external usage by users. All of the extensibility capabilities available to users are also utilized by SymPy itself. This eases the transition pathway from SymPy user to SymPy developer.

The typical way to create a custom SymPy object is to subclass an existing SymPy class, usually Basic, Expr, or Function. As it was stated before, all SymPy classes used for expression trees should be subclasses of the base class Basic. Expr is the Basic subclass for mathematical objects that can be added and multiplied together. The most commonly seen classes in SymPy are subclasses of Expr, including Add, Mul, and Symbol. Instances of Expr typically represent complex numbers, but may also include other "rings", like matrix expressions. Not all SymPy classes are subclasses of Expr. For instance, logic expressions, such as And ( $x, y)$, are subclasses of Basic but not of Expr. ${ }^{15}$

The Function class is a subclass of Expr which makes it easier to define mathematical functions called with arguments. This includes named functions like $\sin (x)$ and $\log (x)$ as well as undefined functions like $f(x)$. Subclasses of Function should define a class method eval, which returns an evaluated value for the function application (usually an instance of some other class, e.g., a Number), or None if for the given arguments it should not be automatically evaluated.

Many SymPy functions perform various evaluations down the expression tree. Classes define their behavior in such functions by defining a relevant eval_* method. For instance, an object can indicate to the diff function how to take the derivative of itself by defining the eval_derivative(self, $x$ ) method, which may in turn call diff on its args. (Subclasses of Function should implement the fdiff method instead; it returns the derivative of the function without considering the chain rule.) The most common eval * methods relate to the assumptions: eval_is_assumption is used to deduce assumption on the object.

Listing 1 presents an example of this extensibility. It gives a stripped down version of the gamma function $\Gamma(x)$ from SymPy. The methods defined allow it to evaluate itself on positive integer arguments, define the real assumption, allow it to be rewritten in terms of factorial (with gamma (x). rewrite(factorial)), and allow it to be differentiated. self. func is used throughout instead of referencing gamma explicitly so that potential subclasses of gamma can reuse the methods.

Listing 1. A minimal implementation of sympy.gamma.

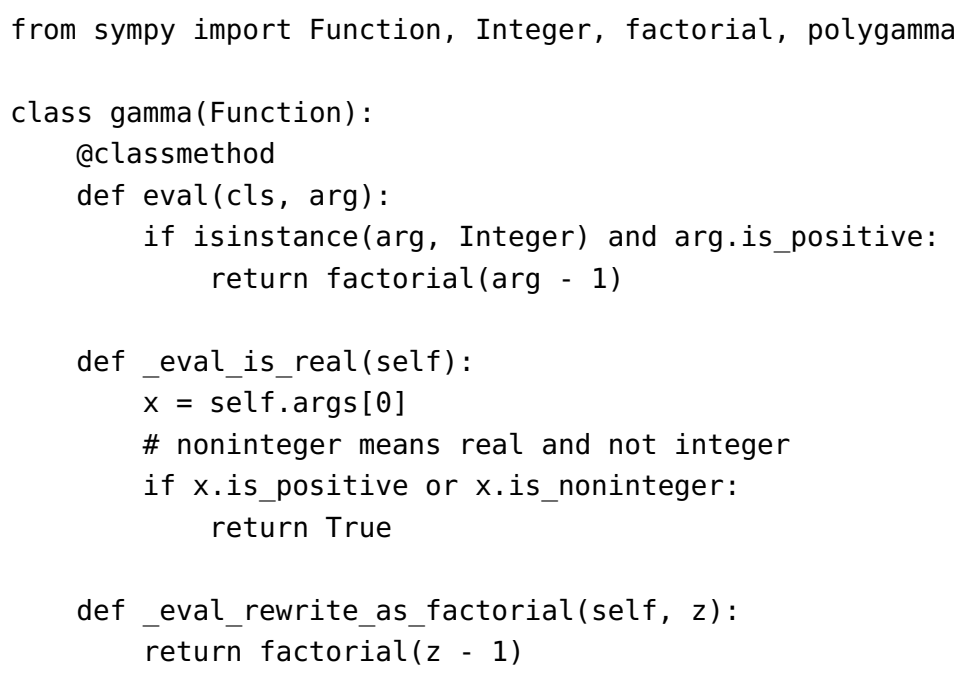




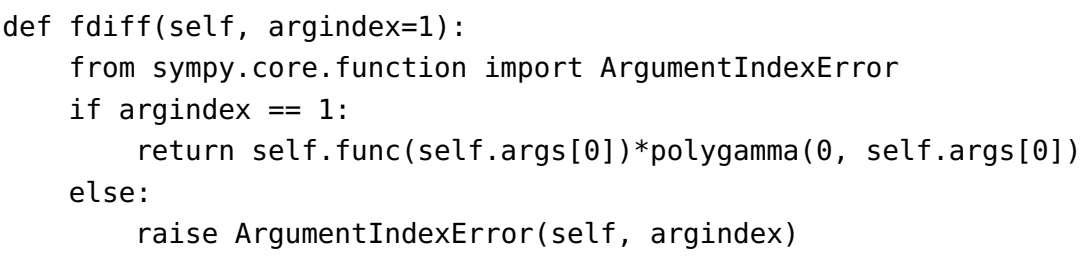

The gamma function implemented in SymPy has many more capabilities than the above listing, such as evaluation at rational points and series expansion.

\subsection{Performance}

Due to being written in pure Python without the use of extension modules, SymPy's performance characteristics are generally poorer than that of its commercial competitors. For many applications, the performance of SymPy, as measured by clock cycles, memory usage, and memory layout, is sufficient. However, the boundaries for when SymPy's pure Python strategy becomes insufficient are when the user requires handling of very long expressions or many small expressions. Where this boundray lies depends on the system at hand, but tends to be within the range of $10^{4}-10^{6}$ symbols for modern computers.

For this reason, a new project called SymEngine [60] has been started. The aim of this poject is to develop a library with better performance characteristics for symbolic manipulation. SymEngine is a pure $\mathrm{C}++$ library, which allows it fine-grained control over the memory layout of expressions. SymEngine has thin wrappers to other languages (Python, Ruby, Julia, etc.). Its aim is to be the fastest symbolic manipulation library. Preliminary benchmarks suggest that SymEngine performs as well as its commercial and open source competitors.

The development version of SymPy has recently started to use SymEngine as an optional backend, initially in sympy.physics.mechanics only. Future work will involve allowing more algorithms in SymPy to use SymEngine as a backend.

\section{PROJECTS THAT DEPEND ON SYMPY}

There are several projects that depend on SymPy as a library for implementing a part of their functionality. A selection of these projects are listed in Table 3.

Table 3. Selected projects that depend on SymPy.

\begin{tabular}{ll}
\hline Project name & Description \\
\hline SymPy Gamma & $\begin{array}{l}\text { An open source analog of Wolfram|Alpha that uses SymPy [61]. There } \\
\text { is more information about SymPy Gamma in section 11 of the supple- } \\
\text { mentary material. }\end{array}$ \\
A CAS designed specifically for the resolution of problems encountered \\
in field theory [39].
\end{tabular}



yt
SfePy
Quameon
Lcapy
A Python package for analyzing and visualizing volumetric data [64]. A Python package for solving partial differential equations (PDEs) in $1 \mathrm{D}, 2 \mathrm{D}$, and $3 \mathrm{D}$ by the finite element (FE) method [66, 10]. Quantum Monte Carlo in Python [57]. An experimental Python package for teaching linear circuit analysis [55].

\section{CONCLUSION AND FUTURE WORK}

SymPy is a robust computer algebra system that provides a wide spectrum of features both in traditional computer algebra and in a plethora of scientific disciplines. It can be used in a first-class way with other Python projects, including the scientific Python stack.

SymPy supports a wide array of mathematical facilities. These include functions for assuming and deducing common mathematical facts, simplifying expressions, performing common calculus operations, manipulating polynomials, pretty printing expressions, solving equations, and representing symbolic matrices. Other supported facilities include discrete math, concrete math, plotting, geometry, statistics, sets, series, vectors, combinatorics, group theory, code generation, tensors, Lie algebras, cryptography, and special functions. SymPy has strong support for arbitrary precision numerics, backed by the mpmath package. Additionally, SymPy contains submodules targeting certain specific physics domains, such as classical mechanics and quantum mechanics. This breadth of domains has been engendered by a strong and vibrant user community. Anecdotally, many of these users chose SymPy because of its ease of access. SymPy is a dependency of many external projects across a wide spectrum of domains.

SymPy expressions are immutable trees of Python objects. Unlike many other CAS's, SymPy is designed to be used in an extensible way: both as an end-user application and as a library. SymPy uses Python both as the internal language and the user language. This permits users to access the same methods used by the library itself in order to extend it for their needs.

Some of the planned future work for SymPy includes work on improving code generation, improvements to the speed of SymPy using SymEngine, improving the assumptions system, and improving the solvers submodule.

\section{REFERENCES}

[1] Adams, W. W. and Loustaunau, P. (1994). An introduction to Gröbner bases. Number 3. American Mathematical Society.

[2] Bailey, D. H., Jeyabalan, K., and Li, X. S. (2005). A comparison of three high-precision quadrature schemes. Experimental Mathematics, 14(3):317-329.

[3] Bender, C. M. and Orszag, S. A. (1999). Advanced Mathematical Methods for Scientists and Engineers. Springer, 1st edition.

[4] Biggs, N., Lloyd, E. K., and Wilson, R. J. (1976). Graph Theory, 1736-1936. Oxford University Press.

[5] Bromborsky, A. (2016). geometric algebra/calculus modules for SymPy. https://github.com/ brombo/galgebra.

[6] Bronstein, M. (2005a). pmint-The Poor Man's Integrator. http://www-sop.inria.fr/cafe/ Manuel. Bronstein/pmint.

[7] Bronstein, M. (2005b). Symbolic Integration I: Transcendental Functions. Springer-Verlag, New York, NY, USA.

[8] Buchberger, B. (1965). Ein Algorithmus zum Auffinden der Basis Elemente des Restklassenrings nach einem nulldimensionalen Polynomideal. PhD thesis, University of Innsbruck, Innsbruck, Austria.

[9] Cervone, D. (2012). Mathjax: a platform for mathematics on the web. Notices of the AMS, $59(2): 312-316$.

[10] Cimrman, R. (2014). SfePy - write your own FE application. In de Buyl, P. and Varoquaux, N., editors, Proceedings of the 6th European Conference on Python in Science (EuroSciPy 2013), pages 65-70. http://arxiv.org/abs/1404.6391.

[11] Ciurana, E. (2009). Google app engine. Developing with Google App Engine, pages 1-10. 
[12] Diankov, R. (2010). Ikfast: The robot kinematics compiler. http://openrave.org/docs/ latest_stable/openravepy/ikfast/.

[13] Doorenbos, R. B. (1995). Production matching for large learning systems. PhD thesis, University of Southern California.

[14] Faugère, J. C. (1999). A New Efficient Algorithm for Computing Gröbner Bases (F4). Journal of Pure and Applied Algebra, 139(1-3):61-88.

[15] Faugère, J. C. (2002). A New Efficient Algorithm for Computing Gröbner Bases Without Reduction To Zero (F5). In ISSAC '02: Proceedings of the 2002 International Symposium on Symbolic and Algebraic Computation, pages 75-83, New York, NY, USA. ACM Press.

[16] Fetter, A. and Walecka, J. (2003). Quantum Theory of Many-Particle Systems. Dover Publications.

[17] Fousse, L., Hanrot, G., Lefèvre, V., Pélissier, P., and Zimmermann, P. (2007). Mpfr: A multiple-precision binary floating-point library with correct rounding. ACM Trans. Math. Softw., 33(2).

[18] Fu, H., Zhong, X., and Zeng, Z. (2006). Automated and Readable Simplification of Trigonometric Expressions. Mathematical and Computer Modelling, 55(11-12):1169-1177.

[19] Gede, G., Peterson, D. L., Nanjangud, A. S., Moore, J. K., and Hubbard, M. (2013). Constrained multibody dynamics with Python: From symbolic equation generation to publication. In ASME 2013 International Design Engineering Technical Conferences and Computers and Information in Engineering Conference, pages V07BT10A051-V07BT10A051. American Society of Mechanical Engineers.

[20] Goldberg, D. (1991). What every computer scientist should know about floating-point arithmetic. ACM Computing Surveys (CSUR), 23(1):5-48.

[21] Gosper, R. W. (1978). Decision procedure for indefinite hypergeometric summation. Proceedings of the National Academy of Sciences, 75(1):40-42.

[22] Gruntz, D. (1996). On Computing Limits in a Symbolic Manipulation System. PhD thesis, Swiss Federal Institute of Technology, Zürich, Switzerland.

[23] Horsen, C. V. (2015). GMPY. https://pypi.python.org/pypi/gmpy2.

[24] Hudak, P. (1998). Domain specific languages. In Salas, P. H., editor, Handbook of Programming Languages, Vol. III: Little Languages and Tools, chapter 3, pages 39-60. MacMillan, Indianapolis.

[25] Hunter, J. D. (2007). Matplotlib: A 2D graphics environment. Computing In Science $\mathcal{E}$ Engineering, 9(3):90-95.

[26] Johansson, F. and The mpmath Development Team (2014). mpmath: a Python library for arbitrary-precision floating-point arithmetic (version 0.19). http://mpmath.org/.

[27] Jones, E., Oliphant, T., Peterson, P., and The SciPy Development Team (2001-). SciPy: Open source scientific tools for Python. [Online; accessed 2016-09-28].

[28] Kane, T. R. and Levinson, D. A. (1985). Dynamics, Theory and Applications. McGraw Hill.

[29] Kluyver, T., Ragan-Kelley, B., Pérez, F., Granger, B., Bussonnier, M., Frederic, J., Kelley, K., Hamrick, J., Grout, J., Corlay, S., Ivanov, P., Avila, D., Abdalla, S., Willing, C., and The Jupyter Development Team (2016). Jupyter notebooks - a publishing format for reproducible computational workflows. In Positioning and Power in Academic Publishing: Players, Agents and Agendas: Proceedings of the 20th International Conference on Electronic Publishing, page 87. IOS Press.

[30] Lagrange, J. (1811). Mécanique analytique. Number v. 1 in Mécanique analytique. Ve Courcier.

[31] Lang, S. (1966). Introduction to transcendental numbers. Reading, Mass.

[32] Lutz, M. (2013). Learning Python. O'Reilly Media, Inc.

[33] Mathpix, Inc. (2016). Mathpix - Solve and graph math using pictures. http://mathpix.com/.

[34] Moses, J. (1971). Algebraic Simplification: A Guide for the Perplexed. In SYMSAC 'r1: Proceedings of the second ACM Symposium on Symbolic and Algebraic Computation, pages 282-304, New York, NY, USA. ACM Press.

[35] Nielsen, M. and Chuang, I. (2011). Quantum Computation and Quantum Information. Cambridge University Press.

[36] Nijenhuis, A. and Wilf, H. S. (1978). Combinatorial Algorithms: For Computers and 
Calculators. Academic Press, New York, NY, USA, second edition.

[37] Oliphant, T. E. (2007). Python for scientific computing. Computing in Science $\&$ Engineering, 9(3):10-20.

[38] Paprocki, M. (2010). Design and implementation issues of a computer algebra system in an interpreted, dynamically typed programming language. Master's thesis, University of Technology of Wrocław, Poland.

[39] Peeters, K. (2007). Cadabra: a field-theory motivated symbolic computer algebra system. Computer Physics Communications.

[40] Pérez, F. and Granger, B. E. (2007). IPython: a system for interactive scientific computing. Computing in Science 83 Engineering, 9(3):21-29.

[41] Peterson, D. L., Gede, G., and Hubbard, M. (2014). Symbolic linearization of equations of motion of constrained multibody systems. Multibody System Dynamics, 33(2):143-161.

[42] Petkovšek, M., Wilf, H. S., and Zeilberger, D. (1997). $A=B$. AK Peters, Ltd., Wellesley, MA, USA.

[43] Raymond, E. (1999). The cathedral and the bazaar. Knowledge, Technology \& Policy, $12(3): 23-49$.

[44] Roach, K. (1996). Hypergeometric function representations. In ISSAC '96: Proceedings of the 1996 International Symposium on Symbolic and Algebraic Computation, pages 301-308, New York, NY, USA. ACM Press.

[45] Roach, K. (1997). Meijer G function representations. In ISSAC '97: Proceedings of the 1997 international symposium on Symbolic and algebraic computation, pages 205-211, New York, NY, USA. ACM.

[46] Rocklin, M. (2013). Mathematically informed linear algebra codes through term rewriting. $\mathrm{PhD}$ thesis, University of Chicago.

[47] Rocklin, M. and Terrel, A. R. (2012). Symbolic statistics with SymPy. Computing in Science and Engineering, 14.

[48] Rose, K. H. (1999). Xy-pic user's guide.

[49] Rosen, L. (2005). Open source licensing, volume 692. Prentice Hall.

[50] Sakurai, J. and Napolitano, J. (2010). Modern Quantum Mechanics. Addison-Wesley.

[51] Shaw, M. and Garlan, D. (1996). Software Architecture: Perspectives on an Emerging Discipline. Prentice Hall. Prentice Hall Ordering Information.

[52] Sussman, G. J. and Wisdom, J. (2013). Functional Differential Geometry. Massachusetts Institute of Technology Press.

[53] Tai, C.-T. (1997). Generalized vector and dyadic analysis: applied mathematics in field theory, volume 9. Wiley-IEEE Press.

[54] Takahasi, H. and Mori, M. (1974). Double exponential formulas for numerical integration. Publications of the Research Institute for Mathematical Sciences, 9(3):721-741.

[55] The Lcapy Developers (2016). Lcapy, a Python package for linear circuit analysis. http: //lcapy.elec. canterbury.ac.nz/.

[56] The Mathics Developers (2016). Mathics, a free, general-purpose online computer algebra system featuring Mathematica-compatible syntax and functions. https://mathics.github.io/.

[57] The Quameon Developers (2016). Quameon, Quantum Monte Carlo in Python. http: //quameon. sourceforge. net/.

[58] The Sage Developers (2016). SageMath, the Sage Mathematics Software System. http: //www. sagemath.org.

[59] The Symbolic Package Developers (2016). The Symbolic Package for GNU Octave. http: //octave. sourceforge. net/symbolic.

[60] The SymPy Developers (2016a). SymEngine, a fast symbolic manipulation library, written in $C++$. https://github.com/symengine/symengine.

[61] The SymPy Developers (2016b). SymPy Gamma. http://www. sympygamma.com/.

[62] The SymPy.jl Developers (2016). SymPy.jl, a package to bring Python's Sympy functionality into Julia via PyCall. https://github.com/JuliaPy/SymPy.jl.

[63] Toth, V. T. (2007). Maple and Meijer's G-function: a numerical instability and a cure. http://www.vttoth. com/CMS/index. php/technical - notes/67.

[64] Turk, M. J., Smith, B. D., Oishi, J. S., Skory, S., Skillman, S. W., Abel, T., and Norman, 
${ }_{858}$ M. L. (2011). yt: A Multi-code Analysis Toolkit for Astrophysical Simulation Data. The 859 Astrophysical Journal Supplement Series, 192:9.

[65] Zare, R. (1991). Angular Momentum: Understanding Spatial Aspects in Chemistry and Physics. Wiley.

[66] Zienkiewicz, O., Taylor, R., and Zhu, J. (2013). The Finite Element Method: Its Basis and Fundamentals. Butterworth-Heinemann, seventh edition edition. 